\title{
Tangence
}

\section{Le regard de Bataille et la pensée de Valéry sous le soleil étoilé de Minuit}

\section{François Ouellet}

Numéro 52, septembre 1996

Tours et détours du romanesque : Minuit aujourd'hui

URI : https://id.erudit.org/iderudit/025914ar

DOI : https://doi.org/10.7202/025914ar

Aller au sommaire du numéro

Éditeur(s)

Tangence

ISSN

0226-9554 (imprimé)

1710-0305 (numérique)

Découvrir la revue

Citer cet article

Ouellet, F. (1996). Le regard de Bataille et la pensée de Valéry sous le soleil

étoilé de Minuit. Tangence, (52), 25-39. https://doi.org/10.7202/025914ar d'utilisation que vous pouvez consulter en ligne.

https://apropos.erudit.org/fr/usagers/politique-dutilisation/ 


\section{Le regard de Bataille et la pensée de Valéry sous le soleil étoilé de Minuit}

\section{François Ouellet}

L'on a dit, à propos des jeunes romanciers de Minuit, qu'ils partageaient peut-être certains traits d'écriture formaliste, comme s'il y avait là l'émergence d'un autre nouveau roman. Est-ce à dire pour autant qu'ils inscrivent leurs démarche en référence à celles des nouveaux romanciers? Dans tous les cas, ils ont "pris acte des leçons du nouveau roman ${ }^{1}$ ", certainement, mais encore? Pour ma part, j'y vois des préoccupations esthétiques qui renverraient, par delà les rapprochements qu'on peut établir entre les nouveaux romanciers de Minuit et Robbe-Grillet, Butor, Simon, Pinget, voire Beckett (chez Toussaint en particulier, par la vélléité des héros et leur relation désœuvrée à la réalité), à un précurseur de l'écriture formelle, Valéry, et à l'une des figures majeures de la modernité, Bataille. Nous y invitent notamment deux romans publiés chez Minuit à l'automne 1986: Monsieur de Toussaint et L'œil de madame de Tropmann, des clins d'œil (c'est le cas de le dire!) respectivement au Monsieur Teste de Valéry et à certains textes de Bataille, en particulier Histoire de l'ceil. Si certains nouveaux romanciers se sont réclámés de Valéry ${ }^{2}$, il apparaît ici que les influences de Toussaint et de Tropmann sont à situer du côté de la revue Tel Quel, dont Valéry a été la première référence et Bataille la référence la plus constante, chez Sollers en particulier ${ }^{3}$,

1 Robert Dion, - Les romanciers de Minuit *, Nuit blancbe, $\mathrm{n}^{\circ} 47$, avril-mai 1992, p. 61 .

2 Claude Simon avec insistance, qui n'a jamais cessé de reconnaître la leçon poétique de Valêry, selon laquelle le poète n'est pas inspiré mais quelqu'un qui fait des choix, car *l'ouvre de l'esprit n'existe qu'en acte - (Paul Valéry, - Première leçon du cours de poẹtique., dans Euvres, t. I, Paris, Gallimard, coll. - Bibliothèque de la Pléiade;, 1968, p. 1349); ne compte dès lors que le faire, le poiein. - Écrire, c'est pour moi, avant tout, faire, résume Simon (Mireille Calle, - L'inclassable réa(e)ncrage du vécu. Entretiens avec Claude Simon, dans Claude Simon. Cbemins de la mémoire, Sainte-Foy, Le griffon d'argile, 1993, p. 3).

3 Il y a aussi ce mot de Denis Roche: *Il y a eu Bataille, il y a Bataille:, cité par Philippe Forest dans Histoire de Tel Quel (1960-1982), Paris, Seuil, 1993. p. 137. 
26

avec Artaud et Ponge. Cela en dépit du fait que ce sont peut-être les contraires qui s'unissent ici, puisque Bataille, selon Leiris ${ }^{4}$, considérait Valéry comme son ennemi numéro un! Il n'est évidemment pas question ici de réduire les textes de Toussaint et de Tropmann à ceux de Valéry et de Bataille, mais, au contraire, de proposer une lecture à partir de ceux-ci, donc d'explorer quelques avenues auxquelles peuvent conduire les multiples voies intertextuelles.

\section{Bataille en trompe-l'œil}

Dans cette nuit opaque, je m'étais rendu ivre de lumière

- Le bleu du ciel

Si Histoire de l'oul de Bataille, .c'est vraiment l'histoire d'un objet $^{5}$ ", L'cil de Madame est. l'histoire d'un objet vivant. L'œil vit, littéralement; il sort la nuit et *bat la campagne", négligeant parfois de rentrer au petit matin, ce qui laisse ceux qui sont privés de leurs yeux dans un état fièvreux et cauchemardesque qui implique un certain érotisme. Madame est la grande prêtresse de cette débauche, et, à ce titre, elle est "la seule sans doute qui [ait] le privilège de l'ôter [son cill et de le remettre à son gré" (p. 75) ${ }^{6}$. L'on se doute déjà que ce roman est, ou doit être, affaire de point de vue. À cet égard, la voix narrative (le discours est assumé par un personnage narrateur anonyme, reçu chez Madame) cédera très vite à l'œil souverain de Madame, qui en quelque sorte assumera la conduite du discours. La fin du premier chapitre donne le ton. Il laisse en plan un récit commencé par Madame à l'adresse du narrateur et se termine sans ponctuation. Madame s'apprêtait à raconter ce qui lui était arrivé alors qu'elle était allée "visiter les mines fabuleuses" de son pays (p. 13). Aucun des autres chapitres du roman ne poursuivra le récit entamé, que ce soit par le sujet ou par des marques grammaticales, comme l'usage de points de suspension ou l'emploi

4 Michel Leiris, - De Bataille l'impossible à l'impossible Documents ${ }^{\circ}$, dans Critique, août-septembre 1963 , p. 686.

5 Roland Barthes, *La métaphore de l'œil *, dans Critique, août-septembre 1963 , p. 770.

6 Tropmann, L'œil de Madame, Paris, Minuit, 1986. Pour les citations subséquentes, la pagination renverra à cette édition. 
d'une minuscule au premier mot d'un chapitre. Or, à l'évidence, la fin du chapitre initial est l'indice d'une orientation thématique et d'une perversion narrative ingénieuse. En effet, c'est bien dans les profondeurs de mines fabuleuses que le narrateur (et le lecteur) sera plongé, appelé dès lors non pas à entendre l'histoire de Madame mais à la vivre. C'est dans la profondeur du fantasme que pénètrera le narrateur, fantasme articulé à la fois sur la base du regard intérieur de l'inconscient et de l'œil scrutateur du voyeur. Cela entraînera la conséquence suivante: l'oeil se substituera rapidement à la parole (celle de Madame aussi bien que celle du narrateur), prenant à śa charge la voix narrative; il en remplit la fonction, il la redouble tout en réduisant au silence le narrateur. Le narrateur fera l'expérience de cette curieuse situation dès le second chapitre, où, Madame ayant quitté le salon, il se retrouve en conversation silencieuse avec l'œil de celle-ci, demeuré "[s]ur le fauteuil, à hauteur de visage", et "animé de toutes les expressions de la vie. (p. 17).

Au fur et à mesure qu'il accumule les expériences, le narrateur devra bien accepter de se ranger sous la domination d'un regard qui tend pourtant à miner son identité: "Il me fallait donc regarder sous une autre lumière tout ce qui m'était arrivé... Ainsi, Madame avait tout conduit... Je me demandai seulement si N... avait été son complice ou si, comme moi.... Ainsi, Madame les menait au fouet "(p. 77) (je souligne). Ce passage traduit parfaitement l'ambiguité que le discours maintient entre l'histoire et sa dérive symbolique, montre combien le discours subordonne la parole au point de vue de Madame. Sans doute elle a conduit son affaire avec une main de fer (elle entretient au fouet des Messieurs en esclavage), mais surtout elle a rondement mené toute cette histoire dans laquelle le narrateur est embarqué malgré lui (la locution indique bien la part obscure qui lève l'inhibition).

Qu'est-ce alors que L'œil de Madame? Un très curieux récit qui est somme toute une seule chose : la représentation d'un imaginaire débridé au sein duquel niche une sexualité morbide, mystique, masochiste. La prolifération et l'exubérance du fantasme annulent toute vraisemblance diégétique et superposent les identités dans ce qui semble être, s'ous l'égide de Madame, une seule façon de vivre le fantasme. Si le narrateur n'a pas plus le contrôle de la narration que des événements qui lui arrivent, c'est qu'il n'est plus, comme les Messieurs, qu'une sorte de sexe à l'affût et au service de Madame. aElle m'ouvrirait les bras - mais quels 
bras! - elle qui m'attendait depuis toujours, depuis le premier jour de ma vie, elle, Madame enfin. (p. 91). Ainsi, les individualités convergent, se superposent, s'emboîtent sous un regard englobant. Sans doute le capitaine N..., dont il est question dans une citation précédente, est-il complice de Madame; quelquefois d'ailleurs il semble mêler sa voix, ou plutôt son regard à celui de Madame. Mais encore, le narrateur ressemble étrangement au capitaine, ce que d'ailleurs Madame est la première à signaler. Le narrateur est au centre de l'aventure, au centre des regards d'autrui parce que le sien est en somme intimement mêlé aux autres. L'extrait suivant est peut-être à cet égard le plus éloquent, d'autant plus qu'il termine un chapitre sans pour autant établir véritablement de lien avec ce qui précédait, conservant une part de flou dans la narration qui rejoint l'atmosphère onirique de tout le roman :

[...] le verre toujours devant moi, et moi ne pouvant y tremper mes lèvres, comme s'il avait été rempli d'une liqueur maléfique, [...] lui ${ }^{7}$, les lèvres minces sur la photographie que j'avais retrouvée, la mâchoire encore durcie, mais les joues rembourrées par l'âge, moi reconnaissant un visage qui me semblait familier, que j'aurais autrefois rencontré dans la rue ou aperçu dans les journaux, un visage qui ne m'était pas inconnu mais que je ne savais à qui attribuer s'il n'était pas le mien; tous paraissait me fixer, ou fixer un point derrière moi (p. 66).

Les Messieurs eux-mêmes sont peut-être le meilleur exemple de cette unicité, de cet amalgame des identités dominé par la présence colossale de Madame. Jadis, dit-on, ils étaient les maîtres, ils étaient puissants (p. 84). Dans leurs cénacles, "ils se tenaient embrassés si fort qu'ils paraissaient ne former qu'un être" (p. 85). Un jour, toutefois, on ne vit plus celui qui "paraissait occuper le milieu de cette masse tassée sur elle-même [...]. Alors la décadence commença. (p. 86). Si aujourd'hui les Messieurs ont été réduits au rang de bêtes par Madame (leur corps est recouvert d'une "espèce de fourruren et ils communiquent par des cris et des *imprécations confuses"), il reste quelque chose de leur unité quand le narrateur les voit pour la première fois, car ils s'emboîtent comme des poupées gigognes (p. 21). Plus encore que dans

7 Nous ne disposons d'aucun point de repère précis pour identifier ce -lui *, qui cependant pourrait très bien être le capitaine; et pas davantage d'indication pour iclentifier le - tous - qui suivra, mais qui renvoie assurément aux Messieurs, qui dévisagent le narrateur d'une pareille façon. 
sa relation à Madame, le narrateur est profondëment tourmenté par les Messieurs, qu'il craint au point d'en faire des cauchemars; ces Messieurs, auxquels il se sent intégré par leur regard enveloppant $^{8}$, sont le reflet décuplé (exacerbé) de l'inconscient, la part instinctuelle à la manifestation de laquelle conduit la rencontre avec Madame.

Assurément, le pseudonyme de l'auteur de L'oeil de Madame fait bien les choses: il est l'homme (man) métaphore (trope), en ce sens que, dans la mesure où nous avons là une écriture de la prolifération, de la métamorphose, du miroir et du reflet, le nom nous informe sur le fonctionnement du texte, un peu comme le titre La jalousie de Robbe-Grillet engendre la fiction; le pseudonyme est ici plus qu'une identité subtilisée, il fonctionne à titre de métaphore structurelle (Ricardou), il est en réalité le signe premier de l'identité fragmentée mais unifiée par la souveraineté irrésistible du désir qui se donne à voir dans le roman. Ce que ce Tropmann indique, n'est-ce pas qu'il jouera à plein la métaphore de l'œil et de sa possession, cet œil que Starobinski n'aurait certainement jamais pensé pouvoir être à ce point *vivant * et qui joue un rôle central dans l'œuvre de Bataille?

Barthes avait montré, à partir de la figure de l'œil dans Histoire de l'oeil, combien l'érotisme de Bataille s'articulait selon la mise en place serrée de chaînes métaphoriques et métonymiques. Il en va autrement chez Tropmann, peut-être parce que l'érotisme reste soigneusement voilé. Bataille, dont le roman est pleinement -pornographique, voire scatologique, choisit de constituer une série d'associations par laquelle l'ouf et le soleil, par exemple, participent du génital ; au contraire, Tropmann déplace cette luminosité de l'œuf et du soleil sur la personne même des Messieurs (ils sont constitués d'une "cavité sombre au milieu du corps avec un point qui brillait ${ }^{9} n$, p. 22), qui dès lors deviennent une sorte de sexe ambulant sans jamais pour autant s'avouer tel. Les scènes érotiques sont rares dans le roman (il y en deux ou trois à la fin),

8 - Il me semblait qu'ils m'enveloppaient * (p. 25), dit-il la première fois que les Messieurs portent leur regard sur lui, formulant une impression qu'il ressentira régulièrement par la suite.

9 Le sexe est ni plus ni moins un oil, comme s'il avait avalé l'objet désirant. L'image nous ramène à une scène centrale de Histotre de l'ail, où Simone glisse un oeil dans son "vagin velu * (Histotre de l'oull, dans CEuvres complètes, t. 1, Paris, Gallimard, 1970, p. 69). 
et pourtant tout le roman les laisse constamment entendre. La transgression du sexe est donnée dans un discours à la fois infiniment plus subtil et complexe, plus littéraire (et en ce sens typique de l'écriture formaliste des romanciers de Minuit) que chez Bataille. Dans Histoire de l'oil, c'est le penseur mystique qui donne dans le roman, alors que dans L'oeil de Madame, c'est un romancier qui joue le jeu d'un certain érotisme, non sans suggérer peut-être un certain mysticisme dans la grandeur de Madame ${ }^{10}$.

Troppmann (avec deux p cette fois) est le pseudonyme que Bataille avait choisi pour W.C., dont il a brûlé le manuscrit, sauf un chapitre, qu'il a gardé comme introduction au Bleu du ciel, dans lequel le protagoniste s'appelle Henri Troppmann. Autre jeu de reflets ${ }^{11}$. Il va sans dire que les références à Bataille sont multiples dans L'oeil de Madame, moins dans le détail que dans la vue d'ensemble. Ici aussi, la littérature est le Mal, qui en est "la valeur souveraine ${ }^{12}$ - non pas le mal, celui qui a pour but l'intérêt (tel crime, par exemple), mais le Mal, celui qui est commis pour jouir, celui qui est l'expression de la transgression, c'est-àdire d'^un désir fou de liberté ${ }^{13}$. Mais la transgression, dans le roman de Tropmann, est sans doute davantage celle d'une écriture ; la sexualité, quant à elle, se manifeste obliquement, souterrainement. L'au-delà de la jouissance que Bataille manifeste par le réseau métaphorique de l'œil, Tropmann le donne à voir par la fonction modale, à laquelle s'intègre le regard fasciné qui épouse le sexe, qui s'y colle en le pénétrant, qui le possède parce qu'il le traverse; c'est ce malaise que ressent le narrateur, qui a l'impression que l'on fixe quelque chose à travers sa personne, devenue le centre d'un * cercle de lumière. (p. 25). L'atteinte du désir est au-delà de l'objet fixé.

Cet au-delà, c'est aussi certainement la mort. Toute l'œuvre de Bataille signifie que l'érotisme est *l'approbation de la vie jus-

10 Je renvoie en particulier au récit de Bataille, Madame Edwarda. : Je sus alors - toute ivresse en moi dissipée - qu'Elle n'avait pas menti, qu'elle était DIEU. (Madame Edwarda, dans Euvres complètes, t. III, Paris, Gallimard, 1971, p. 24).

11 Voir le commentaire de Bataille dans .W.C. Préface à l'Histoire de l'ceil., dans Euvres complètes, t. III, op. cit., p. 57-61. En outre, Bataille avait trouvé son pseudonyme chez un assassin célèbre, Georges Tropmann (avec un p!).

12 Georges Bataille, La littérature et le mal, dans CEutres complètes, t. IX, Paris, Gallimard, 1979, p. 171.

13 Ibid., p. 211. 
que dans la mort ${ }^{14}$. Encore ici, Tropmann ne se limite pas à consigner, comme chez Bataille, la mort de personnages en pleine jouissance; mais il la fait procéder d'une dérive symbolique vers la fin d'un monde, la fin $d u$ monde, celui de l'érotisme effréné de Madame. La mort, dans L'œil de Madame, intervient donc pour mettre fin au récit, qui se termine sur le déclin du pouvoir de Madame à la suite d'une révolte de ses sujets, les Messieurs. Madame et le narrateur s'enfuient en berline et quittent cette existence "pour toujours sans doute. (p. 120), laissant derrière eux un *vieux monde qui s'effondrerait aussitôt comme un château de cartes. (p. 126). Au passage, ils aperçoivent des statues fracassées, "[d]es reines au tronc rompu dont les têtes étaient piquées sur leurs tiges de fer. (p. 120). À l'évidence, cette fin est une caricature de la chute de la monarchie française et plus particulièrement du départ de la famille royale, Madame en tête. Ce n'est pas l'individu jouissant qui meurt ici, mais plus simplement toute cette dimension instinctuelle que la prise en charge d'un regard spéculaire surdéterminé, enivré par ses propres facultés, aura, l'instant d'un roman, mis au jour.

\section{Valéry en trompe-l'esprit ${ }^{15}$}

Là, dans son esprit, régnait toujours la même lumière de nuit.

- Monsieur

Dans les romans de Jean-Philippe Toussaint, au contraire de chez Tropmann, le regard, pivot de multiples variations, n'est pas celui de l'inconscient, mais celui de l'esprit ${ }^{16}$. Et Toussaint accentue suffisamment le trait pour que ses romans - Monsieur en particulier - basculent du côté de Monsieur Teste. Les clins d'œil à Valéry-Teste sont nombreux. Dans La salle de bain, on trouve notamment cette allusion subtile mais sans équivoque, à propos

14. Ibid., p. 174. Cette approbation va jusque dans.l'expérience de la nécrophilie, centrale dans Le bleu du ctel.

15 L'expression est de Picasso, qui insistait par là sur la visée intellectuelle dans la démarche cubiste.

16 Jean-Philippe Toussaint a publié quatre romans, tous publiés aux éditions de Minuit. Comme je les citerai tous, je donne immédiatement les éditions, auxquelles je renverrai dorénavant: La salle de bain (1985), Monsieur (1986), L'apparetl-pboto (1988), La réticence (1991). 
du personnage type de Toussaint, enfermé dans sa pensée, résolument abstrait du monde extérieur: * La souffrance était l'ultime assurance de mon existence, la seule. (p. 95). Dans L'appareilphoto, le narrateur affirme (dans cette syntaxe hachurée particulière à Toussaint) avoir "toujours été assez véloce, d'esprit s'entend, à l'impossible nul n'est tenu • (p. 37). Quant à Monsieur, il conclut que le regard est * une vue de l'esprit " (p. 109). En outre, Monsieur manie le vocabulaire de la physique et des mathématiques, trie méthodiquement ses papiers et annote des articles, suit en pensée le rythme de son propre mouvement (il peut faire cela pendant ades heures égales", p. 29) et travaille "au seizième étage, tour Léonard-de-Vinci (p. 7)! De là à voir dans les personnages de Toussaint, Monsieur en tête! "l'enfant naturel de monsieur Teste ${ }^{17_{n}}$, il n'y a donc qu'un pas, facile à franchir.

Enfant naturel, peut-être; mais dans le saut des générations, il y a forcément quelque chose qui se perd... De fait, à y regarder de près, Toussaint paraît opposer, non sans humour, Monsieur à la figure ambitieuse du père Teste. Teste a voulu atteindre les limites de la pensée humaine, comme Valéry s'est proposé d'imaginer, chez Vinci, * un homme de qui auraient paru des actions tellement distinctes que si je viens à leur supposer une pensée, il n'y en aura pas de plus étendue ${ }^{18}$. L'acuité intellectuelle de Valéry-Teste-Vinci est tout absorbée dans la pensée logique de son propre fonctionnement ou dans celui de l'univers. * [C]'est à l'univers qu'il [Léonard de Vincil songe toujours, et à la rigueur ${ }^{19}$, précise Valéry, qui pareillement revendiquait pour l'expression poétique *le plus de conscience possible ${ }^{20}$. Or, Toussaint prend Valéry à la lettre, inclinant cependant son personnage sur la pente inverse d'une sorte de néantisation spirituelle qui l'exclut de sa propre pensée. Monsieur pense moins pour comprendre que pour s'anéantir. Ayant pris l'habitude d'observer le ciel étoilé, Mónsieur se perd en pensées dans

17 Vincent Landel, * L'enfant naturel de monsieur Teste *, dans Le magazine littératre, $\mathrm{n}^{\circ} 235$, novembre 1986 , p. 58-59.

18 Paul Valéry, Introduction à la métbode de Léonard de Vinci, dans Eưres, t. 1, Paris, Gallimard, coll. • Bibliothèque de la Pléiade ", 1968, p. 1155.

19 Ibid. Référence à la devise de Léonard: Hostinato rigore. Dans La salle de bain, le mot est tourné en dérision: «Rigueur. Le mot me ferait sourire; je tâcherais de ne pas sourire, je ferais demi-tour, marcherais dans les salons une main dans la poche * (p. 29).

20 Paul Valéry, - Fragments des mémoires d'un poème •, dans CEuvres, t. 1, op. cit., p. 1481. 
l'étendue des cieux, l'esprit tendu vers la courbure des horizons. Respirant paisiblement, il parcourait toute la nuit de la pensée, toute, loin dans la mémoire de l'univers, jusqu'au rayonnement du fond du ciel. Atteignant là l'ataraxie, nulle pensée ne se mut plus alors dans l'esprit de Monsieur, mais son esprit était le monde - qu'il avait convoqué (p. 96-97).

Résolument ironique, Toussaint place son personnage en contact avec l'immensité de la connaissance et de la mémoire de l'univers (on sait que Teste a "éduqué. sa mémoire), dans laquelle se dissout la pensée de celui qui paraît réaliser la position exemplaire de l'inconsistance, de l'absence, de la perte - celle du nom sans aucun doute. En effet, Monsieur vit dans la pensée, mais bors des exigences d'une saisie rigoureuse de la connaissance ${ }^{21}$. Il est un Monsieur sans la tête qui caractérise le patronyme du héros de Valéry. Monsieur se possède et possède le monde dans une sorte d'absence de soi, ce qui contrarie et abolit le programme de Teste, qu'il rejoint pourtant dans le partage des mêmes conditions d'expérience; seul le résultat change, mais c'est beaucoup. En fait, ce que Monsieur expérimente, c'est ni plus ni moins l'expérience de la mort de Teste (à la lettre la disparition du nom) ou ala marche funèbre de la pensée "22, quand le regard du penseur dérive "hors de ce monde, œil frontière entre l'être et le non-être" qui établit une sorte de degré zéro de la pensée. "Tout à l'heure, peut-être, avant d'en finir, j'aurai cet instant important - et peut-être me tiendrai-je tout entier dans un coup d'œil terrible ${ }_{n}^{23}$.

Malgré la jouissance qu'ils peuvent trouver à épuiser la réalité par la mécanique intellectuelle, il semble donc que les personnages de Toussaint tendent davantage vers cette mort - immobilité et absence - de l'esprit, vers l'acquisition d'une sorte de position foetale ${ }^{24}$ qui n'est pas sans rappeler Beckett. Je pense notamment

21 Dans L'appareil-photo: :L'endroit où je me trouvais s'était peu à peu dissipé de ma conscience et je fus un instant idéalement nulle part, si ce n'est immobile dans mon esprit . (p. 102).

22 Paul Valéry, «Fin de monsieur Teste•, dans Monsieur Teste, dans CEuvres, t. II, Paris, Gallimard, coll. * Bibliothèque de la pléiade *, 1960, p. 75.

23 Ibid., p. 74.

24 Voir cette scène de L'apparetl-pboto où le personnage se tient sur le pont d'un bateau au coeur de la nuit. "Aucune terre ne se dessinait encore à l'horizon, le ciel était immense dans la nuit et la mer elle-même semblait s'être étendue aux cieux. Parfois, oui, la mort me manquait* (p. 105-106) (je souligne). Le choix du verbe est révélateur d'un état antérieur à retrouver. Voir aussi la citation que je donne plus loin de la page 113 de L'appareil-pboto. 
34

à Murphy renversé sur une chaise, les membres noués, et dont *[1]'esprit [...] s'imagin[e] comme une grande sphère creuse, fermée hermétiquement à l'univers extérieur " ${ }^{25}$. Tiraillés entre une impuissance à s'inscrire dans la réalité et un penchant irrésistible au "désespoir d'être" de la pensée abstraite ${ }^{26}$, les personnages de Toussaint substituent à la réalité extérieure - une réalité tout autre, intérieure et docile "sans mots ni sons mais seulement constituée "des formes en mouvements qui suiv[ent] leur cours dans [l']esprit comme le mouvement même du temps, avec la même évidence infinie et sereine", dit le narrateur de L'appareilphoto (p. 125). Le temps est ici mouvement - de l'esprit -, mais sera ultimement, conformément aux citations précédentes, arrêt sur la jouissance de la pensée autarcique. Dans chaque roman, le personnage essaie "de se représenter que le mouvement, aussi fulgurant soit-il en apparence, tend essentiellement vers l'immobilité, et qu'en conséquence, aussi lent peut-il parfois sembler, entraîne continûment les corps vers la mort, qui est immobilité " (La salle de bain, p. 36).

"Le mouvement son caractère relatif" (p. 76), c'est exactement ce que Monsieur enseigne aussi au jeune Ludovic. Sur ce plan, l'humour de Monsieur est particulièrement éclatant par rapport à la pensée valérienne. Chez Teste, le regard, foncièrement dynamique, est tout dévoué à la compréhension des mécanismes de l'esprit. De la même façon que ce n'était pas le poème qui intéressait Valéry, mais l'acte qui le produisait, Teste s'intéresse à ce qui lui est extérieur pour l'assimiler à sa propre connaissance - conscience lucide. "En résumé, je me sens être dans ses mains, entre ses pensées, comme un objet qui tantôt lui est le plus familier, tantôt le plus étrange du monde, selon le genre de son regard variable qui s'y adapte ${ }^{27}$, écrit madame Émilie Teste au sujet de son mari. Le regard est variable à la fois dans son mouvement et sa portée subjective, parce que c'est l'ensemble des conditions de la pensée que Teste cherche à saisir. Le travail de l'esprit fait qu'il est «l'être absorbé dans sa variation, celui qui devient son système, celui qui se livre tout entier à la discipline

25 Samuel Beckett, Murpby, Paris, Minuit, 1990, p. 81.

26 Le narrateur de L'apparell-pboto caractérise l'extraction de la pensée du monde de la pierre au profit de son propre monde intérieur par le passage progressif : de la difficulté de vivre au désespoir d'être * (p. 94). - Lettre de madame Émilie Teste., dans Monsieur Teste, op. cit., p. 31. 
effrayante de l'esprit libre ${ }^{28} n$. Or, si Valéry-Teste multiplie les regards afin de cerner la complexité de la mécanique, c'est précisément ce relativisme qui empêche Monsieur d'agir et l'immobilise. "C'est selon" est une formule privilégiée par Monsieur, qui résume l'interprétation de Copenhague, selon laquelle le regard pouvait "altér[er] de façon radicale la description mathématique" de l'état d'un animal, *le faisant passer de l'état de limbes à un nouvel état, où il était soit positivement en vie, soit positivement mort, c'était selon" (p. 27). Or, Monsieur agit, dans la vie quotidienne, suivant la leçon tirée de l'interprétation de Copenhague. Par exemple, au restaurant avec Anna Bruckhardt, dont il est amoureux sans toutefois savoir comment la courtiser, Monsieur tente de parer à son hésitation en demandant à Anna si elle préfère être invitée ou partager l'addition! Mais Anna n'a pas de préférence, ce qui plonge à nouveau Monsieur dans l'embarras. "Monsieur lui confia qu'il n'avait aucune idée de ce qu'il convenait de faire dans ces cas-là. [...] Qu'est-ce qu'on fait, alors? dit Monsieur et, baissant la tête, il se plongea dans la contemplation de ses doigts dans l'obscurité (p. 107-108). Le trait est grossi, mais tout à fait représentatif de la loi physique citée par Monsieur et à laquelle il est implacablement soumis, voué en bout de ligne à une inertie à peu près totale, se constituant lui-même *comme une hypothèse physique " ${ }^{29}$. Le libre-arbitre? pensez-vous...

À mon avis, Monsieur étêté est la figure la plus éloquente de l'esthétique de Toussaint. En conformité avec cette abstraction du monde extérieur au profit d'une position intérieure - mais dont la jouissance est donnée pour elle-même, sans la mesure de la règle et de la connaissance - qui signe l'absence d'identité, avec cette soumission à un relativisme qui paralyse la démarche intellectuelle et la retourne contre elle-même, il y a la mise en place d'une optique narrative contaminée par défaut peut-être,

28 -La soirée avec monsieur Teste*, dans Monsieur Teste, op. cit., p. 18.

29 Bien que différemment de Léonard, dont c'est l'immense génie qui l'apparente à une -hypothèse physique. (Introduction à la méthode de Léonard de Vinci, op. cit., p. 1175). Vincent Landel croit dénoter comme trait commun à Toussaint, Echenoz et Deville une esthétique • fondée sur la.science et sur les réalités de l'ordre cellulaire, transposée dans l'ordre humain* (* Le démon de Deville , dans Le magazine littéraire, $\mathrm{n}^{\circ} 257$, septembre 1988, p. 76). Deville place au cour de son - livre scientifique. Longue vue la physique de Maxwell, lui-même cité par Valéry dans son Introduction comme l'un des chercheurs ayant eu l'intuition de la méthode qu'il propose. 
construite à même l'absence. Le plus bel exemple - mais le plus complexe serait dans La réticence - est celui qu'offre L'appareilphoto. La photographie idéale du narrateur serait celle qui témoignerait d'un "élan furieux" hors de la réalité : "la photo serait floue mais immobile, le mouvement serait arrêté, rien ne bougerait plus, ni ma présence ni mon absence, il y aurait là toute l'étendue de l'immobilité qui précède la vie et toute celle qui la suit, à peine plus lointaine que le ciel que j'avais sous les yeux" (p. 113). Du reste, aucune des photos qu'il aura prises ne sera tirée, la pellicule laissant apparaître "ça et là quelques ombres informes comme d'imperceptibles traces de [s]on absence" (p. 116). La difficulté du personnage de s'ancrer dans la vie fait donc corps avec une structure narrative elle-même incertaine, dont le narrateur est incapable d'assumer la focalisation au sens fort du terme, c'est-à-dire d'orienter et de déterminer une intrigue qui puisse avoir quelque consistance et soutenir la voix narrative, réduite à ne consigner que de l'insignifiance.

Avec Monsieur, Toussaint est allé au plus court. Valéry a signalé les correspondances du nom de son personnage avec la tête et l'œil témoin: "Conscious - Teste, Testis ${ }^{30}$. Décapité, Monsieur perdait la profondeur du regard. Cette absence, Monsieur, qui préfère se déplacer dans Paris en convertissant dans son esprit le ciel étoilé en "un gigantesque plan de métro illuminant la nuit" (p. 93), la prolonge physiquement. Dans les réunions de la compagnie pour laquelle il travaille, Monsieur "veill[e] scrupuleusement à rester dans l'axe [du] corps [de madame DuboisLacour], reculant lorsqu'elle recul[e], avançant lorsqu'elle se pench[e] en avant, de manière à n'être jamais trop directement exposén (p. 12). Il est vrai que Teste aussi s'efface: "Tout s'effaçait en lui, les yeux, les mains ${ }^{31}$. Mais Monsieur s'éclipse parce qu'il est simplement un être terne, l'effacement est la condition de sa présence au monde. Toussaint radicalise, systématise l'attitude de Teste - comme Monsieur contrefait donc la position foetale de la pensée vers laquelle il gravite au petit hasard de la fatalité.

C'est peut-être par sa position intellectuelle et l'impuissance qui le caractérise que Monsieur, figure nominale exemplaire de

30 - Pour un portrait de monsieur Teste-, dans Monsieur Teste, op. cit., p. 64.

31 . La soirée avec monsieur Teste *, dans Monsieur Teste, op. cit., p. 17. 
l'anonymat, incarne le mieux cette *ère du vide" (Lipovetsky) par laquelle la littérature peut se dire postmoderne et, non pas formuler une fin de l'histoire, mais refléter "le dernier des hommes" annoncé par Nietzsche, "l'homme de la technologie froide et des affects morcelés [...], de l'exil intérieur, [s]chizoïde hors des murs de l'hôpital psychiatrique ${ }^{32}$ ". Teste lui-même disait déjà : "Je ne suis pas tourné du côté du monde. J'ai le visage vers le MUR. Pas un rien de la surface du mur qui me soit inconnu ${ }^{33}$ ". La reconfiguration du héros romanesque participe largement, du reste, du renouvellement esthétique désiré et généreusement inauguré par le Valéry de Tel quel. En voulant gommer le personnage au profit de la construction formelle qui le constitue, lui et son univers de papier, Valéry annonce, bien malgré lui, et bien au-delà des personnages anonymes de Sarraute et de l'initiale à laquelle est réduite l'héroine de La jalousie, la dépersonnalisation qui peutêtre caractérise les nouveaux héros de chez Minuit dans leur rapport au monde, et, en particulier, l'insignifiance exemplaire des héros de Toussaint ${ }^{34}$.

32 Roland Jaccard, L'exil intérieur. Scbizotide et civilisation, Paris, Seuil, coll. "Points *, 1978, p. 15.

33 "Quelques pensées de monsieur Teste., dans Monsteur Teste, op. cit, p. 72. Comment ne pas penser aux personnages de Toussaint qui s'intéressent longuement aux lézardes des murs? * Le mur qui me faisait face, parsemé de grumeaux, présentait des craquelures; des cratères ça et là trouaient la peinture terne. Une fissure semblait gagner du terrain. Pendant des heures, je guettais ses extrêmités, essayant vainement de surprendre un progrès" (La salle de bain, p. 12).

34 On rapprochera des romans de Toussaint ceux d'Éric Chevillard. La nébuleuse du crabe (Paris; Minuit, 1993), par exemple, met en scène un personnage éminemment beckettien et kafkaïen, flou, "comme à la merci d'un coup de gomme" (p. 86) et sans * fonction- (*pour remplir sa fonction, quelle fonction, et comment procéder, et par où commencer, commencer quoi?", p. 10), qui désire une seule chose: - se clébarrasser de la conscience "(p. 101), c'està-dire *simplement ne plus avoir de tête [...] pour la brandir au bout d'une pique et la promener ainsi dans les rues * (p. 110). On trouve dans ce roman l'essentiel des figures de l'absence des romans de Toussaint, depuis le vide de l'autoportrait photographique («une photo ratée, floue, tremblée*, p. 51), en passant par la perte du regard concomitante à celle de la tête $(\cdot$ Crab est insaisissable, ni fuyant ni dérobé, plutôt flou, comme si sa myopie native avait peu à peu rongé tous ses tissus •, p. 7), jusqu'à l'anéantissement dans le cosmos ( . Ou peut-il s'en remettre entièrement à sa seule force de concentration - jusqu'à griller ses lumières par excès de tension? Conscience dure et trop lucide, étoile fine, pointue, piquante, perçante, pénétrante, qui coud la nuit sur le jour - désintégrée tout à coup, explosée, répandue, apaisée: naissance d'une nébuleuse*, p. 12). On voit que, comme chez Toussaint, le 
Insignifiance, inconsistance, qui semble dès lors être la réplique tout aussi virulente que défaitiste que propose cette littérature. On connaît la question centrale qui obsédait Teste: "Que peut un homme? ${ }^{35}$ ". Question posée avant lui par Dostö̈evski et Nietzsche et à laquelle ont tenté de répondre les écrivains de la première moitié du vingtième siècle, qu'il s'agisse d'Apollinaire, de Malraux, de Saint-Exupéry ou de Gide (je ferais le pari qu'on dresserait sans peine une histoire de la littérature moderne à partir de cette seule question). Or, Monsieur ne peut rien, il est absolument incapable de la moindre initiative. "Monsieur, oui, en toutes choses, son mol acharnement" (p. 60). Et l'adjectif est un euphémisme. Depuis Valéry, il y a eu Freud et Einstein, comment l'oublier ici... Un anti-héros, donc, Monsieur, mais une possibilité de héros fin-de-siècle qui incarne, somme toute, la seule forme d'affirmation de soi légitime - "comme ces lutteurs orientaux qui désarment l'adversaire par leur apparente faiblesse "36, pour reprendre une formule d'Yvan Leclerc dans un article sur les romans de Toussaint. À cet égard - ultime pirouette Toussaint ne fait pas que subvertir la position intellectuelle valéryenne, mais il lui substitue la validité d'une autre, dont l'hérö̈sme tient à la vacuité qui la caractérise. Paradoxalement, Monsieur, déjouant, piégeant la rhétorique du narrateur de Monsieur Teste, devient celui que "la niaise manie de son nom" ne possède pas ${ }^{37}$.

Ce qui reste, dans tous les cas, c'est une position pour le moins inconfortable. C'est à la fois une réticence extrême à s'inscrire dans la réalité et un réflexe de survie qui rattache ultimement la pensée - qui compose avec la mort - au monde exté-

personnage cle Chevillard, né - avec le cerveau à la place du coxur * (p. 85), pense puissamment mais pour retourner la pensée contre elle-même.

- La soirée avec monsieur Teste , dans Monsieur Teste, op. cit., p. 23.

36 Yvan Leclerc, *Abstraction faite*, Critique, $\mathrm{n}^{\circ} 510$, novembre 1989, p. 897.

37 "La soirée avec monsieur Teste., dans Monsteur Teste, op. cit., p. 15. "Chaque esprit qu'on trouve puissant, commence par la faute qui le fait connâitre- (p. 16), écrivait Valéry. S'oppose alors à Monsieur le personnage de Kaltz, minéralogiste et attaché de recherches au CNlRS. Il dicte à Monsieur, qui ne sait pas lui refuser son concours, son ouvrage sur la cristallographie, dont plusieurs extraits, cités comme tels dans le roman, sont d'un niveau de difficulté extrême pour le lecteur qui n'est pas spécialiste. Il y a là un effet recherché que met en relief sa contre-partie, c'est-à-dire le discours de Monsieur sur la relativité (voir par exemple le contraste avec les propos de Kaltz de la page 65). 
rieur, geste désespéré plutôt que conquérant. Ambiguité - relativisme? - qui s'exprime le mieux peut-être dans La réticence précisément, où le personnage vacille entre le désir de son effacement le plus complet (résistance à visiter son ami à qui il s'était pourtant annoncé) et le désir de se rendre présent à l'autre (certitude qu'il est épié par son ami). Il vit sur la marge, la frontière, entre deux chaises, profondément réticent, signe d'une ambivalence que consignait déjà Monsieur, plaçant habilement le mot à la faveur d'une permutation métonymique: "Monsieur, une chaise. Là, entre deux réticences, il tâchait de se réfugier dans la pratique apaisante de gestes simples* (p. 89). La fin des romans est tout de même explicite quant au désir du personnage de conjurer la mort, de survivre à sa propre pensée (à la lettre!): l'un, prenant "le risque de compromettre la quiétude de [s]a vie abstraite ", quittera sa baignoire (La salle de bain, p. 123), l'autre tentera de saisir l'instant présent d'une aube magnifique qui lui redonne la vie (le mot "vivant" est le dernier de L'appareil-photo et est isolé par un blanc), le narrateur de La réticence se laissera gagner par le bruit de la mer qui lui apporte *comme un soulagement des sens et de l'esprit" et s'ancrera les pieds "dans le sol pour [s]'imprégner toujours plus de la sensation de bien-être que [lui] procur[e] le contact du sable mouillé. (p. 158); et Monsieur, après lui avoir expliqué la théorie des quanta d'Ilya Prigogine, prendra la main d'Anna Bruckhardt. 